\title{
Smith-Magenis syndrome in monozygotic twin fetuses presenting with discordant phenotypes and uteroplacental insufficiency
}

\author{
YI ZHOU $^{1 *}$, YINGJUN XIE $^{1 *}$, YUNXIAO ZHU $^{2}$, JIANZHU WU ${ }^{1}$, \\ MEIJIAO SHANG ${ }^{1}$, BAOJIANG CHEN ${ }^{1}$ and QUN FANG $^{1}$ \\ Departments of ${ }^{1}$ Obstetrics and Gynaecology and ${ }^{2}$ Ultrasonic Medicine, Fetal Medicine Center, \\ The First Affiliated Hospital of Sun Yat-sen University, Guangzhou, Guangdong 510080, P.R. China
}

Received December 21, 2014; Accepted October 14, 2015

DOI: $10.3892 / \mathrm{mmr} .2015 .4538$

\begin{abstract}
Smith-Magenis syndrome (SMS) is a rare condition with multiple congenital malformations caused by the haploinsufficiency of RAI1 (deletion or mutation of RAI1). However, the correlation between genotype and phenotype is not well understood. The present study describes the prenatal diagnosis of monozygotic twins with a $17 \mathrm{p} 11.2$ deletion, which is indicative of SMS, who presented with discordant phenotypes and uteroplacental insufficiency. A high-resolution genome-wide single nucleotide polymorphism array revealed a $3.7-\mathrm{Mb}$ deletion in the $17 \mathrm{p} 11.2$ chromosome region. Accurate breakpoints of the deletion in these patients were used to identify correlations between SMS and the concomitant phenotypes, particularly uteroplacental insufficiency, which has rarely been investigated in SMS. In addition, no exonic mutations were identified in or affected known disease-associated loci that could explain the congenital anomalies, according to a model that accounts for the possibility of incomplete penetrance. Furthermore, a novel benign copy number variation (a duplication of $195 \mathrm{~kb}$ at 13q12.13) was identified but was unlikely to be clinically significant in the discordant phenotypes of the twins. The present study showed that multiple interacting genetic and environmental factors are involved in determining the variance of the SMS phenotype.
\end{abstract}

\section{Introduction}

Smith-Magenis Syndrome (SMS) is a rare neurodevelopmental disorder caused by a microdeletion on chromosome $17 \mathrm{p} 11.2$ and has an incidence of $\sim 1$ in 25,000 live births (1).

Correspondence to: Professor Qun Fang, Department of Obstetrics and Gynaecology, Fetal Medicine Center, The First Affiliated Hospital of Sun Yat-sen University, 58 Zhongshan No. 2 Road, Guangzhou, Guangdong 510080, P.R. China

E-mail: fangqun2014@sina.com

*Contributed equally

Key words: Smith-Magenis syndrome, high-resolution single nucleotide polymorphism, discordant phenotypes, exome sequencing
Individuals with SMS exhibit wide phenotypic variability. Certain patients show mild to moderate mental retardation, while others exhibit atypical facial features, and cardiac, renal and otolaryngologic abnormalities (1-3). However, the correlation between genotype and phenotype is not well understood in SMS (3-5).

For a number of decades, monozygotic (MZ) twin comparisons have been used to identify the contributions of nature (heredity) and nurture (environment) (6). Recently, several studies have suggested that genetic and epigenetic factors exhibit a role in phenotypic variance $(2,3,7)$. Phenotypic variability is often observed between unrelated or related individuals or twins with the same microdeletion syndrome, such as 22q11.2 (3,8-10). Observation of the genetic history of a syndrome in MZs often leads to a greater understanding of the phenotypic variability (11). Recently, a high resolution single nucleotide polymorphism (SNP)-array allowed for the detection of copy number variants (CNVs) as well as SNPs. Halder et al (12) reported a case in which twins carrying the 22q11.2DS microdeletion had discordant phenotypes with a different sized genetic deletion. Another technique, exome sequencing, offers an efficient and affordable method to investigate the genetic factors involved in human diseases (13-16). B.D. Solomon performed exome sequencing on MZs discordant for VACTERL (vertebral anomalies, anal atresia, cardiac malformations, tracheo-esophageal fistula, renal anomalies and limb abnormalities) association-type congenital malformations (17). It was hypothesized that this method may reveal discordant variants that are able to explain the cause(s) of disease (17). Hicks et al (11) reported a case of MZs with SMS with different phenotypes; however, further molecular investigation of the discordant phenotypes was not conducted. The standard study design in these cases is to use current molecular techniques, such as an SNP array and exome sequencing, in order to identify correlations between SMS and the concomitant phenotypes, in addition to investigating the factor that may contribute to the phenotypic variability in MZs with SMS. In the present study, current molecular techniques, including as an SNP array and exome sequencing, were used un order to identify correlations between SMS and the concomitant phenotypes, in addition to investigating the factors, which may contribute to the phenotypic variability in MZs with SMS 


\section{Patients and methods}

Clinical description. A 24-year-old woman, gravida 3, para 1, with a monochorionic diamniotic twin pregnancy was referred to the prenatal department of the Department of Obstetrics and Gynaecology, The First Affiliated Hospital of of Sun Yat-Sen University (Guangzhou, China) at 28 weeks and 4 days gestation. The patient presented with the presence of a ventricular septal defect and stenosis of the main pulmonary artery in one twin; the other twin was normal. Her two previous pregnancies were uncomplicated, and the third pregnancy was conceived spontaneously with a 25 -year-old man. Ultrasound examination (GE Voluson 730 Expert; GE Healthcare Life Sciences, Vienna, Austria) at the cessation of menstruation, at 9 weeks, and at 16 weeks revealed that the twins were developmentally delayed by 2 weeks. These ultrasound findings and her delayed menstrual cycle postponed the expected delivery date by 2 weeks. A thin dividing membrane between the fetuses was visualized on ultrasound. The twins (Fig. 1A) were diagnosed with fetal growth retardation (FGR) at 28 weeks and 4 days gestation due to the 2 -week developmental delay. A sonographic examination suggested measurable growth discordance. In addition, one twin (twin 1) was observed to have a ventricular septal defect $(3 \mathrm{~mm})$ and stenosis of the main pulmonary artery (Fig. 1C), while the other twin (twin 2), with the exception of FGR showed no abnormalities (Fig. 1B). The amniotic fluid volume (AFV) of twin 1 and twin 2 was $48 \mathrm{~mm}^{3}$ and $31 \mathrm{~mm}^{3}$, respectively. Following genetic counseling, an ultrasound-guided amniocentesis of the fetuses was performed for a cytogenetic analysis and chromosomal microarray analysis (CMA) at 29 weeks and 4 days gestation. The ultrasound examination of the AFV of twin 1 and 2 prior to cordocentesis were 13 and $21 \mathrm{~mm}^{3}$, respectively.

One week following surgery, the AFV of the two fetuses recovered to normal with volumes of 37 and $28 \mathrm{~mm}^{3}$, in twins 1 and 2, respectively, but the ventricular septal defect was enlarged to $3.5 \mathrm{~mm}$ in twin 1 . Additionally, twin 1 developed a more severe form of FGR and absent end-diastolic flow of the umbilical artery. The ultrasound testing performed 2 weeks after amniocentesis revealed oligohydramnion of both fetuses, with AFVs of $9.7 \mathrm{~mm}^{3}$ (twin 1) and $12 \mathrm{~mm}^{3}$ (twin 2). Doppler investigations (GE Voluson 730 Expert; GE Healthcare Life Sciences) of the umbilical arteries and ductus venosus revealed no abnormal findings. Analysis of the amniocentesis sample by CMA revealed a 17p11.2 deletion, which was characteristic of SMS. The patient was called back for additional genetic counseling to discuss the results and prognosis of both fetuses. The patient decided to continue the pregnancy after multiple discussions.

Ultrasound examination at 34 weeks and 3 days gestation revealed the intrauterine fetal death of both twins and the absence of amniotic fluid. The two fetuses were delivered by stillbirth at the Obstetric Department of The First Affiliated Hospital of Sun Yat-Sen University). Both fetuses were visually nearly normal. However, organ malformations could not be determined as the parents did not consent to a fetal autopsy.

Ethical approval was obtained for this study from the Ethics Committee of the First Affiliated Hospital of Sun Yat-Sen University. All data were collected with the informed consent of the patient.
Cytogenetic analysis. Routine cytogenetic analysis using G-banding techniques at a resolution of 550 bands was performed. Briefly, a $10 \mathrm{ml}$ sample of amniotic fluid was collected and subjected to amniocyte culture according to the standard cytogenetic protocol (18). A $5 \mathrm{ml}$ sample of parental blood was collected from each parent and subjected to lymphocyte culture according to the standard blood cytogenetic protocol (18).

Whole-genome high-resolution SNP array. CMA on the uncultured amniotic fluid was performed using an Affymetrix cyto HD Array (Affymetrix, High Wycombe, UK). DNA was amplified, labeled, and hybridized to a CytoScan HD array platform, according to the manufacturer's protocol. The array was designed specifically for cytogenetics research and offers more than two million markers across the genome, including SNP probes and probes to detect copy number variations (Cyto-arrays). CEL files, obtained by scanning the CytoScan arrays, were analyzed with Chromosome Analysis Suite software (Affymetrix) using the annotations of genome version GRCH37 (hg19). Only those achieving the manufacturer's quality cut-off measures (MAPD $\leq 0.25$; SNP QC $\geq 15.0$; waviness standard deviation $\leq 0.12$ ) were included in the analysis. Gains and losses that affected a minimum of 50 markers over a 100-kbp length were initially considered. Changes in copy number were compared with the CNVs catalogued in the Database of Genomic Variants (DGV; http://dgv.tcag.ca/dgv/ app/home) and the University of Santa Clara in California (UCSC; http://genome.ucsc.edu/) genome browser. The gene content of the CNVs of interest was determined using the UCSC browser, based on the Genome Reference Consortium Human Genome (GRCH; build 37; http://genome.ucsc.edu/).

Exome sequencing. The zygosity of the twins was confirmed using small tandem repeat markers. Polymorphic DNA marker analysis was performed on the parental and fetal DNAs using an ABI Prism 3500 (Applied Biosystems, Foster City, CA, USA). Solution hybridization exome capture was performed using the SureSelect Human All Exon v5 systems (Agilent Technologies, Santa Clara, CA, USA) using biotinylated RNA baits to hybridize sequences that corresponded with exons (19). The manufacturer's software version 1.5 , which is compatible with Illumina paired-end sequencing software (Illumina, San Diego, CA, USA), was used. The manufacturer's specifications for the v5 kit report that the capture regions total $\sim 50 \mathrm{Mb}$, which corresponded with the Consensus Conserved Domain Sequences database (http://www.sanger.ac.uk/resources/databases/encode/) that contains $>1,000$ non-coding RNAs as well as Gencode Project defined exons. Targeted regions included the exons of 18,113 genes of the Consensus Conserved Domain Sequences database, exons of additional genes, miRNAs, and non-coding RNA genes, totaling 30,241 genomic features and $51,646,629$ targeted bases. Flowcell preparation and sequencing were performed according to the protocol for the HiSeq 2000 sequencer (Illumina) using 100-bp paired-end reads to generate sufficient data such that $\geq 85 \%$ of the targeted bases were accurately genotyped, with an average coverage of 150x. Image analysis and base calling were performed on all data lanes using the Illumina HiSeq Control Software (HCS) v2.0.5 and RTA software v1.17.20 (Illumina) with default parameters. 
A

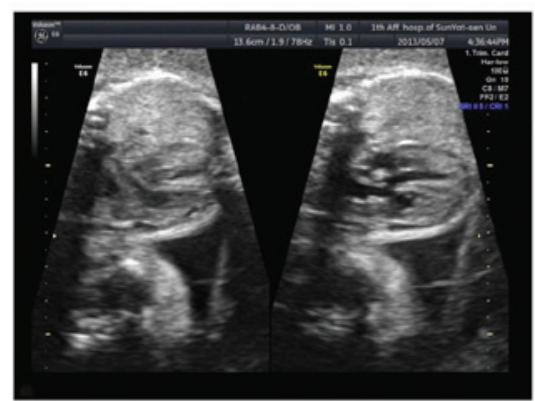

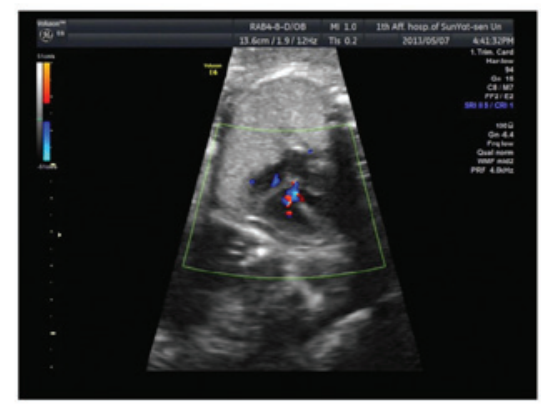

C

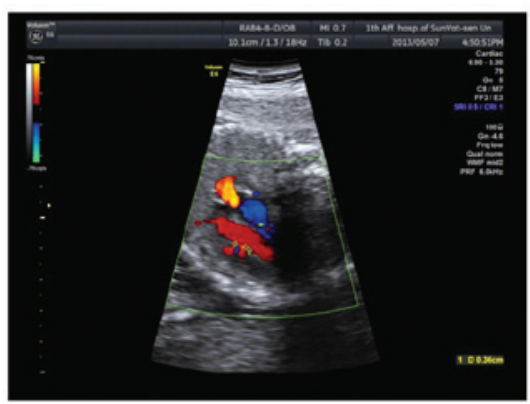

D

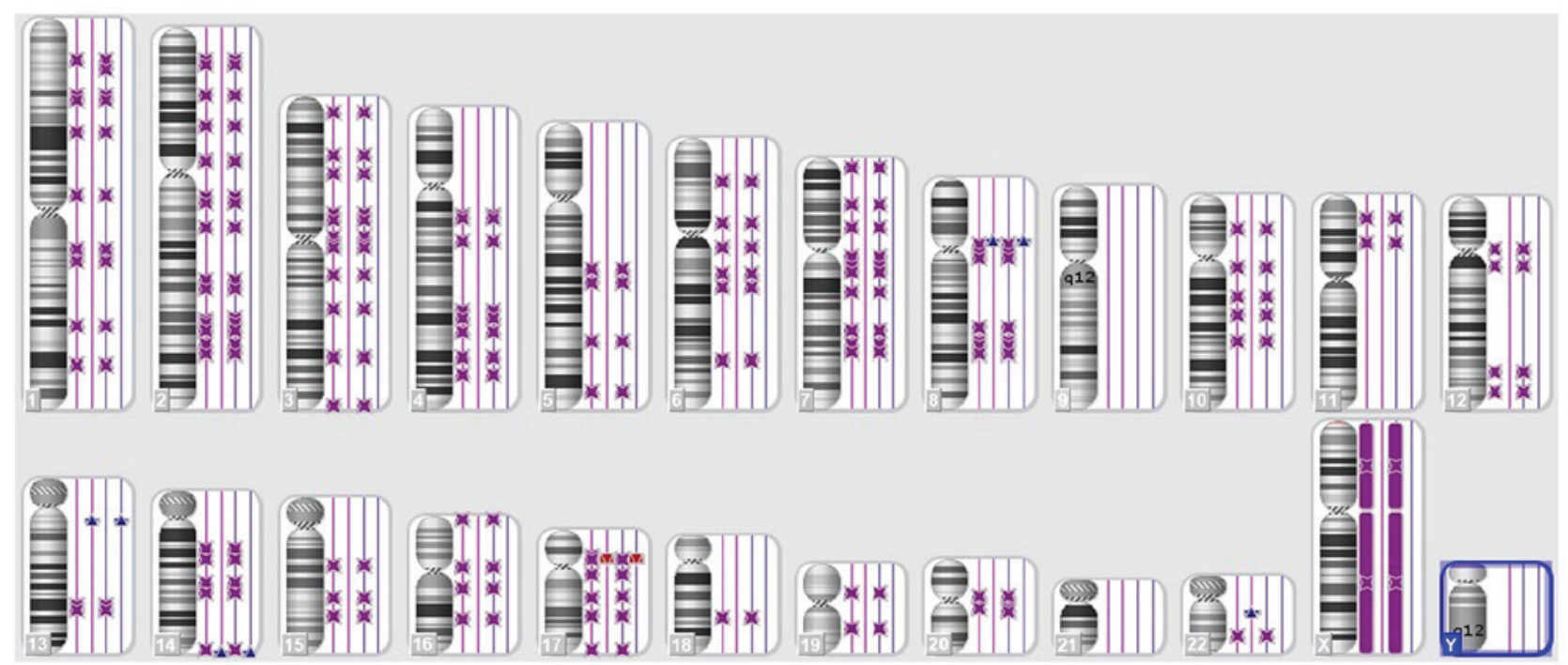

$\mathbf{E}$

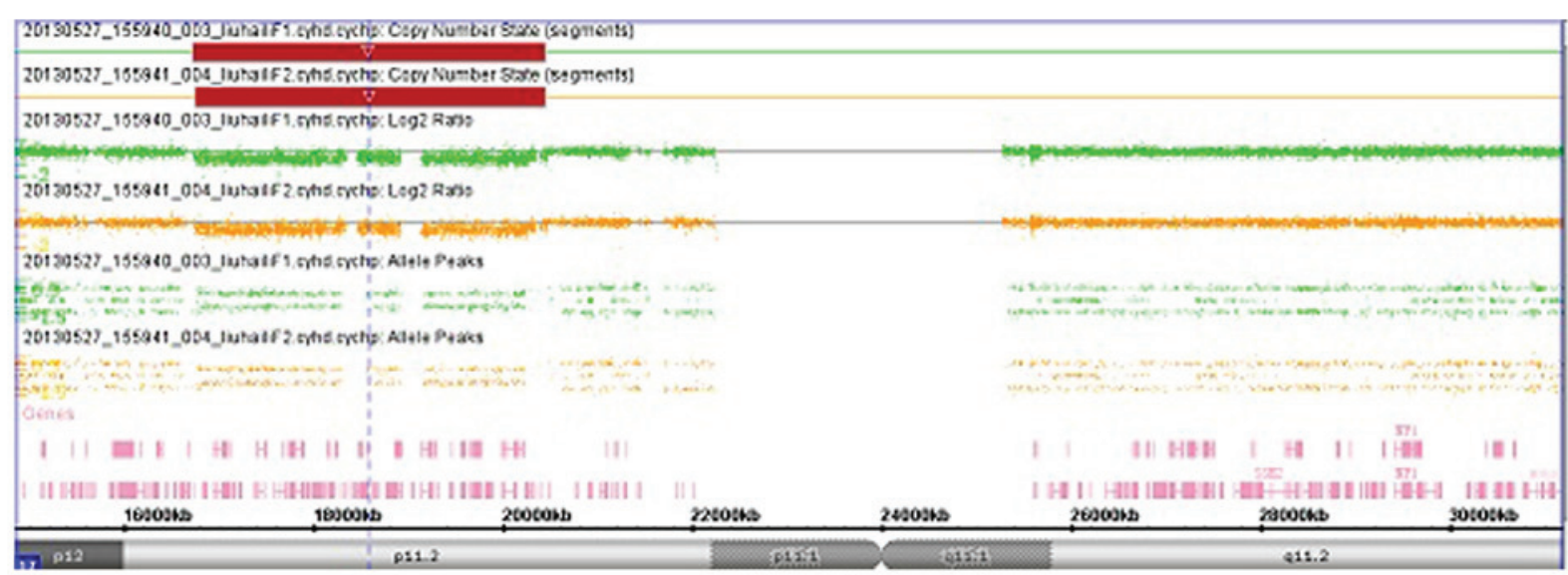

Figure 1. (A) Sonographic examination of both twins. (B) Sonographic examination of twin 1. (C) Sonographic examination of twin 2. (D) Long-contiguous stretch of homozygosity patterns observed in the twins (twin 1 and twin 2, respectively) shown in the purple star. CNV patterns are shown in the red (loss) and blue (gain) boxes. (E) Microdeletion at 17p11.2. The microarray profile of the $17 \mathrm{p} 11.2$ microdeletion is represented by the bold red line. The schematic diagram is shown below, and the deleted region is shown by a red box (twin 1 and twin 2, respectively).

\section{Results}

Chromosome analysis. The chromosome analysis revealed a normal male karyotype in both fetuses. The parents also had a normal karyotype. However, the CMA indicated that the fetuses had a deletion at chromosome 17 as arr $17 \mathrm{p} 11.2(16$, $761,814-20,433,502) \times 1$ in one fetus, and, arr 17p11.2 (16, 761, $814-20,433,502) x 1$ in the other fetus. Both harbored the RAI1 gene, which is a critical gene involved in SMS (Fig. 1E). The parents had a normal CMA result, indicating a de novo deletion in both fetuses.

$C N V$ identification. With the exception of the $17 \mathrm{p} 11.2$ deletion, the CMA identified a total of 9 CNVs. The identified $\mathrm{CNV}$ sere classified as being absent in the DG) or present in the DGV (Table I). The sizes of these CNVs vary from 
Table I. Identity of copy number variants in the family members.

\begin{tabular}{|c|c|c|c|c|c|}
\hline $\begin{array}{l}\text { Family } \\
\text { member }\end{array}$ & CNVs & Size $(\mathrm{kb})$ & Gene & Type & Inherited \\
\hline \multirow[t]{5}{*}{ Father } & $13 \mathrm{q} 12.13(26,803,491-26,918,775) \times 3$ & 115 & CDK8 & Novel & \\
\hline & $14 q 32.33(106,072,250-106,728,149) \times 3$ & 656 & KIAA0125, ADAM6 & $\mathrm{PD}$ & \\
\hline & $16 p 11.2(33,596,761-33,774,726) \times 1$ & 178 & & PD & \\
\hline & $19 \mathrm{p} 12(20,588,836-20,716,153) \times 1$ & 127 & ZNF826P & $\mathrm{PD}$ & \\
\hline & $22 \mathrm{q} 11.22(23,063,020-23,258,369) \times 3$ & 195 & MIR650, IGLL5 & $\mathrm{PD}$ & \\
\hline \multirow[t]{4}{*}{ Mother } & $1 \mathrm{p} 11.2(121,225,582-121,339,317) \times 3$ & 114 & EMBP1 & $\mathrm{PD}$ & \\
\hline & 12 p13.31 $(8,006,510-8,124,048) \times 3$ & 118 & SLC2A14, SLC2A3 & PD & \\
\hline & 19 p12 $(20,588,836-20,720,705) \times 1$ & 132 & ZNF826P & $\mathrm{PD}$ & \\
\hline & $14 q 32.33(106,251,147-106,728,149) \times 3$ & 477 & KIAA0125, ADAM6 & PD & \\
\hline \multirow[t]{3}{*}{ Twin 1} & $13 q 12.13(26,803,491-26,918,933) \times 3$ & 115 & CDK8 & Novel & pat \\
\hline & 8 p11.22 $(39,247,097-39,386,952) \times 3$ & 140 & ADAM5P, ADAM3A & PD & $\mathrm{dn}$ \\
\hline & $14 q 32.33(106,072,264-106,728,149) \times 3$ & 656 & KIAA0125, ADAM6 & PD & pat \\
\hline \multirow[t]{4}{*}{ Twin 2} & $13 q 12.13(26,803,491-26,927,389) \times 3$ & 124 & $\mathrm{CDK} 8$ & Novel & pat \\
\hline & 8p11.22 $(39,254,032-39,384,337) \times 3$ & 130 & ADAM5P, ADAM3A & PD & $\mathrm{dn}$ \\
\hline & $14 q 32.33(106,072,264-106,728,149) \times 3$ & 656 & KIAA0125, ADAM6 & $\mathrm{PD}$ & pat \\
\hline & $22 q 11.22(23,063,020-23,258,369) \times 3$ & 195 & MIR650, IGLL5 & $\mathrm{PD}$ & pat \\
\hline
\end{tabular}

CNVs, copy number variants; PD, present in the database of genomic variants; novel, absent in the database of genomic variants; pat, paternal; $\mathrm{dn}$, de novo.

114 to $656 \mathrm{~kb}$. In the fetuses, the duplication at $13 \mathrm{q} 12.31$ was inherited from the father, and the other CNV at 8p11.22 was a de novo event. Exome sequencing revealed the same disorder in the twins. A polymorphic DNA marker showed that the twins were a perfect match monozygosity (data not provided) and confirmed the twin pairs by high-density SNP microarray analysis (Fig. 1D). In brief, neither microarray analysis nor exome sequencing revealed an obvious discordant genetic anomaly (CNV or exome mutation) that would readily explain the presence of the congenital anomalies in one of the twins.

\section{Discussion}

The present study reports a pair of MZtwins, with a 3.7 Mb microdeletion at $17 \mathrm{p} 11.2$. This type of deletion is 'common' as $70 \%$ of patients with SMS have an $\sim 3.7 \mathrm{Mb}$ microdeletion in the 17p11.2 region (https://decipher.sanger.ac.uk/syndrome/8\#overview). However, the twin pair presented with discordant phenotypes: One with a ventricular septal defect and stenosis of the main pulmonary artery, and the other as nearly normal.

The majority of SMS features are due to an RAI1 haploinsufficiency (20), while the variability and severity of the disorder are modified by other genes in the $17 \mathrm{p} 11.2$ region. The functional role of RAI1 is not completely understood, but based on homology and preliminary studies, it is likely to be involved in transcription $(21,22)$. A phenotypic comparison between patients with deletions and patients with RAI1 mutations shows that 21 out of 30 SMS features are the result of an RAI1 haploinsufficiency, whereas cardiac anomalies, speech and motor delay, hypotonia, short stature and hearing loss are associated with 17 p11.2 deletions rather than RAI1 mutations (7). In addition to RAI1, the $3.7 \mathrm{Mb}$ region deleted in the present case contains at least 50 genes, including mitogen-activated protein kinase 7 (MAPK7) activated by an upstream cascade of kinases in response to a wide variety of extracellular stimuli. Specific PRKM kinases (MAPKKs or PRKMKs) have been shown to phosphorylate and activate specific PRKMs in a given signaling pathway. Hayashi et al (23) concluded that the MAPK7 pathway is critical for endothelial function and the maintenance of blood vessel integrity. In addition, a common cause of intrauterine growth restriction in humans is uteroplacental vascular insufficiency, which increases the incidence of perinatal asphyxia and neurodevelopmental disorders (24). A recent study showed that the cardiac dimensions are spared and may be used for gestational age estimation in growth-restricted fetuses resulting from uteroplacental insufficiency (25). A case report of MZ twins with SMS also shows both fetuses presenting with FGR (11), which is concordant with the present study. However, whether a fetus with SMS is more prone to FGR and whether haploinsufficiency of the PRKM7 gene is connected with uteroplacental insufficiency requires further investigation.

The twins also had a duplication at $13 \mathrm{q} 12.31$ which was inherited from the father. It was absent in the DGV and there was no reported $\mathrm{CNV}$ in this region. The $\mathrm{CNV}$ only included one protein-encoding gene CDK8 (cyclin-dependent kinases $8, * 603184)$, which encodes a member of the mediator complex, located at 13q12.13, a region of recurrent copy number gain in a substantial fraction of colon cancers (26). Spore investigation confirmed the importance of Cdk8 at multiple stages of Dictyostelium development, although the severity of the defect in spore production depends on the genetic background (27). Although, it is important to consider the possibility of incomplete penetrance, the family did not know of any history of cancer. CDK8 is also a haploinsufficiency 
gene in DECIPHER (28). While, deletions in the region have clinical relevance, duplication of the same interval may be benign (29). The present study also used phenotype databases, such as OMIM or gene/mutation-specific databases included in the specific $\mathrm{CNV}$, and, considering the normal phenotype of the father, the duplication at $13 \mathrm{q} 12.31$ appears to be a benign polymorphism in the family and did not affect the twins SMS-associated features.

The zygosity of the twins, first confirmed by a polymorphic DNA marker and genomic DNA analysis on a high-resolution SNP array, showed the twins were a perfect match, except for the size of the CNV. However, except for experimental error and its proximal endpoint located in a low copy number, repeat or segmental duplication region, as evidenced on the UCSC genome browser (hg19), no CNV or exonic mutations were identified or affected known disease-associated loci that would explain the congenital anomalies for the possibility of incomplete penetrance. In this study, a high-resolution SNP array and exome sequencing of the MZ but phenotypically discordant twins did not explain why only one member of the twin pair was affected with features associated with SMS. There are multiple possible explanations: The presence of a regulatory factor that affects gene expression or a coding-region $\mathrm{CNV}$, the presence of mutations only in the affected individual, or the mutations occurred in a region that may not be revealed by current methods of CNV analysis. Eventually, developments in genome testing may be able to evaluate these hypotheses, and there may be a discordant mutation not detected by the applied CNV. Microarray studies were conducted on DNA extracted from amniotic fluid, and similar testing based on other tissue types may yield greater success, as has recently been demonstrated in Proteus syndrome (30). The occurrence of a twin pregnancy may act on susceptible alleles to result in congenital malformations. Although parental studies were performed in this twin pair, molecular studies indicated that there were variants present in the parents but not the $17 \mathrm{p} 11.2$ deletion. Additionally, other testing modalities, such as methylation analysis, in conjunction with $\mathrm{CNV}$, may shed more light on disease pathogenesis. Finally, it is possible that the causes of these congenital malformations may not be directly gene-related and may involve a primary, currently unidentified, environmental factor. Finally, on a level more specific to SMS, it is likely that multiple interacting genetic and environmental factors are involved in determining phenotype.

Further studies are ongoing related to several genetic variants of high interest (not located in the genes previously shown to be associated with human disease) that were found in the two twins and that may act in concert as susceptibility factors.

\section{Acknowledgements}

The authors would like to thank the staff of the Prenatal Center (Department of Obstetrics and Gynaecology, The First Affiliated Hospital of Sun Yat-Sen University) for providing patient data.

\section{References}

1. Smith ACM, Boyd KE, Elsea SH, et al: Smith-Magenis syndrome In: Genereviews. Pagon RA, Adam MP, Bird TD, Dolan CR, Fong CT and Stephens K (eds.), Seattle (WA), 1993.
2. Madduri N, Peters SU, Voigt RG, Llorente AM, Lupski JR and Potocki L: Cognitive and adaptive behavior profiles in Smith-Magenis syndrome. J Dev Behav Pediatr 27: 188-192, 2006.

3. Andrieux J, Villenet C, Quief S, Lignon S, Geffroy S, Roumier C, de Leersnyder H, de Blois MC, Manouvrier S, Delobel B, et al: Genotype phenotype correlation of 30 patients with Smith-Magenis syndrome (SMS) using comparative genome hybridisation array: Cleft palate in SMS is associated with larger deletions. J Med Genet 44: 537-540, 2007.

4. Gamba BF, Vieira GH, Souza DH, Monteiro FF, Lorenzini JJ, Carvalho DR and Morreti-Ferreira D: Smith-Magenis syndrome: Clinical evaluation in seven Brazilian patients. Genet Mol Res 10: 2664-2670, 2011.

5. De Leersnyder H: Smith-Magenis syndrome. Handb Clin Neurol 111: 295-296, 2013.

6. Hall JG: Twinning. Lancet 362: 735-743, 2003.

7. Girirajan S, Vlangos CN, Szomju BB, Edelman E, Trevors CD, Dupuis L, Nezarati M, Bunyan DJ and Elsea SH: Genotype-phenotype correlation in Smith-Magenis syndrome: Evidence that multiple genes in $17 \mathrm{p} 11.2$ contribute to the clinical spectrum. Genet Med 8: 417-427, 2006.

8. Singh SM, Murphy B and O'Reilly R: Monozygotic twins with chromosome 22q11 deletion and discordant phenotypes: Updates with an epigenetic hypothesis. J Med Genet 39: e71, 2002.

9. Desmaze C, Scambler P, Prieur M, Halford S, Sidi D, Le Deist F and Aurias A: Routine diagnosis of DiGeorge syndrome by fluorescent in situ hybridization. Hum Genet 90: 663-665, 1993.

10. Van Hemel JO, Schaap C, Van Opstal D, Mulder MP, Niermeijer MF and Meijers JH: Recurrence of DiGeorge syndrome: Prenatal detection by FISH of a molecular 22q11 deletion. J Med Genet 32: 657-658, 1995.

11. Hicks M, Ferguson S, Bernier F and Lemay JF: A case report of monozygotic twins with Smith-Magenis syndrome. J Dev Behav Pediatr 29: 42-46, 2008.

12. Halder A, Jain M, Chaudhary I and Varma B: Chromosome 22q11.2 microdeletion in monozygotic twins with discordant phenotype and deletion size. Mol Cytogenet 5: 13, 2012.

13. Fahiminiya S, Almuriekhi M, Nawaz Z, Staffa A, Lepage P, Ali R, Hashim L, Schwartzentruber J, Abu Khadija K, Zaineddin $\mathrm{S}$, et al: Whole exome sequencing unravels disease-causing genes in consanguineous families in Qatar. Clin Genet 86: 134-141, 2014.

14. Kono M, Sugiura K, Suganuma M, Hayashi M, Takama H, Suzuki T, Matsunaga K, Tomita Y and Akiyama M: Whole-exome sequencing identifies ADAM10 mutations as a cause of reticulate acropigmentation of Kitamura, a clinical entity distinct from Dowling-Degos disease. Hum Mol Genet 22: 3524-3533, 2013.

15. Nuytemans K, BademciG, Inchausti V, Dressen A, Kinnamon DD, Mehta A, Wang L, Züchner S, Beecham GW, Martin ER, et al: Whole exome sequencing of rare variants in EIF4G1 and VPS35 in Parkinson disease. Neurology 80: 982-989, 2013.

16. Ravenscroft G, Thompson EM, Todd EJ, Yau KS, Kresoje N, Sivadorai P, Friend K, Riley K, Manton ND, Blumbergs P, et al: Whole exome sequencing in foetal akinesia expands the genotype-phenotype spectrum of GBE1 glycogen storage disease mutations. Neuromuscul Disord 23: 165-169, 2013.

17. Solomon BD, Hadley DW, Pineda-Alvarez DE, Kamat A, Teer JK, Cherukuri PF, Hansen NF, Cruz P, Young AC, Berkman BE, et al; NISC Comparative Sequencing Program: Incidental medical information in whole-exome sequencing. Pediatrics 129: e1605-e1611, 2012.

18. Verma R and Babu A (eds): Human Chromosomes: Principles \& Techniques, 2nd edition. McGraw-Hill, New York, NY, p419, 1995 [book review]. Mol Reprod Devel 43: 134, 1996.

19. Gnirke A, Melnikov A, Maguire J, Rogov P, LeProust EM, Brockman W, Fennell T, Giannoukos G, Fisher S, Russ C, et al: Solution hybrid selection with ultra-long oligonucleotides for massively parallel targeted sequencing. Nat Biotechnol 27: 182-189, 2009.

20. Carmona-Mora P, Canales CP, Cao L, Perez IC, Srivastava AK, Young JI and Walz K: RAI1 transcription factor activity is impaired in mutants associated with Smith-Magenis Syndrome. PLoS One 7: e45155, 2012.

21. Lacaria M, Gu W and Lupski JR: Circadian abnormalities in mouse models of Smith-Magenis syndrome: Evidence for involvement of RAI1. Am J Med Genet A 161A: 1561-1568, 2013.

22. Girirajan S, Elsas LJ II, Devriendt K and Elsea SH: RAI1 variations in Smith-Magenis syndrome patients without 17p11.2 deletions. J Med Genet 42: 820-828, 2005. 
23. Hayashi M, Kim SW, Imanaka-Yoshida K, Yoshida T, Abel ED, Eliceiri B, Yang Y, Ulevitch RJ and Lee JD: Targeted deletion of BMK1/ERK5 in adult mice perturbs vascular integrity and leads to endothelial failure. J Clin Invest 113: 1138-1148, 2004.

24. Burke C, Sinclair K, Cowin G, Rose S, Pat B, Gobe G and Colditz P: Intrauterine growth restriction due to uteroplacental vascular insufficiency leads to increased hypoxia-induced cerebral apoptosis in newborn piglets. Brain Res 1098: 19-25, 2006.

25. Uerpairojkit B, Manotaya S, Tanawattanacharoen S, Wuttikon sammakit $\mathrm{P}$ and Charoenvidhya D: Are the cardiac dimensions spared in growth-restricted fetuses resulting from uteroplacental insufficiency? J Obstet Gynaecol Res 38: 390-395, 2012.

26. Firestein R, Bass AJ, Kim SY, Dunn IF, Silver SJ, Guney I, Freed E, Ligon AH, Vena N, Ogino S, et al: CDK8 is a colorectal cancer oncogene that regulates beta-catenin activity. Nature 455 : $547-551,2008$
27. Greene DM, Bloomfield G, Skelton J, Ivens A and Pears CJ: Targets downstream of Cdk8 in Dictyostelium development. BMC Dev Biol 11: 2, 2011.

28. Huang N, Lee I, Marcotte EM and Hurles ME: Characterising and predicting haploinsufficiency in the human genome. PLoS Genet 6: e1001154,2010.

29. Grayton HM, Fernandes C, Rujescu D and Collier DA: Copy number variations in neurodevelopmental disorders. Prog Neurobiol 99: 81-91, 2012.

30. Lindhurst MJ, Sapp JC, Teer JK, Johnston JJ, Finn EM, Peters K, Turner J, Cannons JL, Bick D, Blakemore L, et al: A mosaic activating mutation in AKT1 associated with the Proteus syndrome. N Engl J Med 365: 611-619, 2011. 